Document downloaded from:

http://hdl.handle.net/10251/66689

This paper must be cited as:

Mohamed Mohamed-Hicho, N.; Antonino Daviu, E.; Cabedo Fabres, M.; Ferrando Bataller, M. (2015). A novel low-profile high-gain UHF antenna using high-impedance surfaces. IEEE Antennas and Wireless Propagation Letters. 14:1014-1017. doi:10.1109/LAWP.2015.2389274.

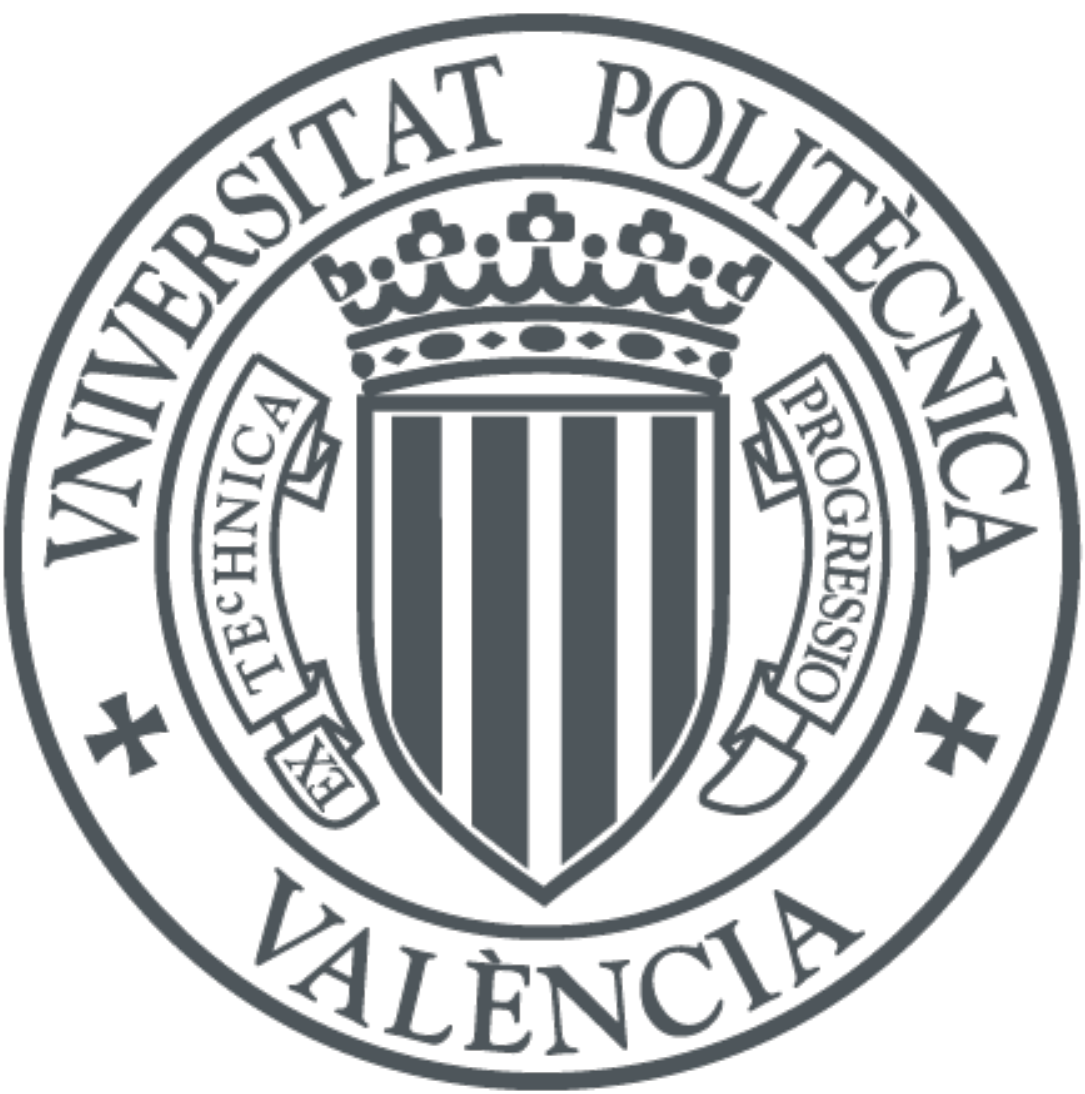

The final publication is available at

http://dx.doi.org/10.1109/LAWP.2015.2389274

Copyright Institute of Electrical and Electronics Engineers (IEEE)

Additional Information 


\title{
A Novel Low-Profile High-Gain UHF Antenna Using High-Impedance Surfaces
}

\author{
Nora Mohamed Mohamed-Hicho,Student Member, IEEE, Eva AntoninoDaviu, Member, IEEE, Marta \\ Cabedo-Fabrés, Member, IEEE, Miguel FerrandoBataller, Member, IEEE
}

\begin{abstract}
A novel wideband low-profile planar antenna design based on the use of a High-Impedance Surface (HIS) is presented for Digital Television (DTV) reception in the UHF band. The proposed design is based on a wideband monopole antenna in close proximity to an artificial ground plane, composed of an array of square metal loops at the top, an air gap and a ground plane at the bottom, without grounded vias. Low-cost substrates are employed in the design: rigid PVC for the antenna, and foamed PVC for the artificial ground plane. The advantages of the proposed design for DTV applications are high gain, low profile, light weight, low cost and large bandwidth. Details of the proposed antenna design are described, and measurements together with a comparison to a conventional antenna with PEC ground plane are presented.
\end{abstract}

Index Terms-Artificial magnetic conductor (AMC), electromagnetic band-gap (EBG), UHF antenna, low-profile antenna, high-gain, DTV antenna, HIS.

\section{INTRODUCTION}

$\mathrm{H}$ IGH Impedance Surfaces (HIS) are of special interest for antenna design due to two remarkable properties: First, they can behave as perfect Artificial Magnetic Conductors (AMC), what enables efficient radiation for antennas placed parallel and close to the surface; second, they forbid the propagation of electromagnetic waves in certain frequency band (EBG), so that there is an absence of surface waves and radiation patterns are smoother [1]-[3].

Wideband antennas with High Impedance Surfaces (HIS) have been investigated in the last years to provide low-profile antennas with a simple structure, large bandwidth and increased gain [1]-[11]. By using an AMC or a periodic structure to replace the conventional PEC reflector, unidirectional radiation can be obtained for a wide variety of antennas [4]-[11].

Digital Television (DTV) applications at UHF band require antennas covering a large frequency band, ranging from 470 $\mathrm{MHz}$ to $790 \mathrm{MHz}$. This means a $50.8 \%$ bandwidth for an antenna covering this application, where unidirectional radiation pattern is also required. Little investigation has been

Manuscript received. This work was supported by Spanish Ministry of Science and Innovation under the projects TEC2010-20841-C04-01, TEC2013-47360-C3-3-P and CSD2008-00068.

N. Mohamed Mohamed-Hicho, E. Antonino-Daviu, M.Cabedo-Fabrés and M. Ferrando-Bataller are with the Institute of Telecommunications and

Multimedia Applications (iTEAM), Edificio 8G, UniversitatPolitècnica de València, Camino de Vera s/n, 46022, Valencia, Spain (email: nomomo@upvnet.upv.es, evanda@upvnet.upv.es). done up to now relating EBG or AMC antennas for DTV applications or UHF band.

In [11], a wideband bowtie dipole in close proximity to an EBG ground plane was presented for the UHF band, covering from $309 \mathrm{MHz}$ to $520 \mathrm{MHz}$. Although impedance bandwidth was not limited by the use of the EBG ground plane, a certain degradation of the radiation patterns was observed at lower and upper frequencies of the operating band. Moreover, the final design of the structure was too heavy and unfeasible for practical applications, because of the use of conventional EBG ground planes. Therefore, other solutions are needed to reduce the overall weight as well as fabrication costs for these antennas.

In this paper, a novel UHF planar antenna operating over a frequency range from $470 \mathrm{MHz}$ to $790 \mathrm{MHz}$ is presented. The proposed antenna combines a basic wideband planar monopole antenna with a HIS structure. In order to provide unidirectional radiation behavior and reduce the height of the antenna, a HIS structure is used as the reflecting surface [4][11]. In this way, the antenna can be placed very close to the reflector. A low-cost, environment-friendly and long-lasting dielectric substrate is proposed for both the antenna and the HIS in order to reduce costs.

A HIS structure composed of a Frequency Selective Surface (FSS) [12][13], an air gap and a ground plane is proposed to reduce the height and weight of the overall structure, while maintaining the wideband behavior required for this UHF application. The novelty of the paper is thus the proposal of a unidirectional antenna for UHF band with $55.6 \%$ relative bandwidth, high-gain $(10.25 \mathrm{dBi})$, low-profile (height $<0.1 \lambda$ ), low-cost (by using commercial low-cost substrates) and light weight (due to a new HIS structure). Therefore, the proposed antenna constitutes an efficient and practical solution for DTV reception applications.

The antenna geometry and its design process will be described next, and measured results for a fabricated prototype will be presented later, showing the excellent behavior of the proposed design. Finally, an equivalent circuit model for the HIS structure will be presented.

\section{II.LOW-PROFILE ANTENNA OVER TRADITIONAL HIS}

This section presents the characteristics of a planar wideband monopole antenna over a conventional 3x4 planar HIS structure, used as a reflector. The geometry of the HIS unit cell, formed by a square loop, and its dimensions are 
shown in Fig. 1. This HIS geometry is chosen because it offers wide bandwidth characteristics [8]. The initial unit cell is $110 \times 110 \mathrm{~mm}^{2}$, and it is printed on a $10 \mathrm{~mm}$ thick foamed PVC material with a dielectric constant of 2.4 and low density, around $0.55-0.74 \mathrm{gr} / \mathrm{cm}^{3}$. There are no vias to the ground plane, in order to simplify the design.

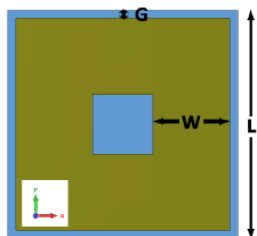

(a)

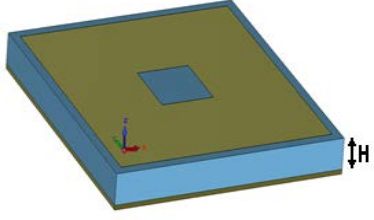

(b)
Fig. 1. (a) Initial unit cell physical dimensions (in $\mathrm{mm}$ ): $\mathrm{L}=110 ; \mathrm{W}=38.5$; $\mathrm{G}=4$; (b) Lateral view: $\mathrm{H}=10 \mathrm{~mm}$.

AMC and EBG properties of this HIS structure were analyzed in [14]. Moreover the unit-cell geometry was optimized using CST Microwave Studio 2012. A single cell with Periodic Boundary Conditions (PBC) was simulated to model the reflection phase characteristics, in order to search for the AMC in-phase band (which is from $351 \mathrm{MHz}$ to 793 MHz) [14]. The HIS structure can also act as an EBG structure by exhibiting frequency band gaps, which in this case occur close to the AMC operating frequency band (from $100 \mathrm{MHz}$ to $715 \mathrm{MHz}$ ) [14]. The number of HIS unit-cells has an important influence on the antenna radiation performance. Consequently, analysis of surface currents in a large HIS plane shows that the surface currents are located close to the radiating element. Thus the size of the HIS plane can be reduced to $3 \times 4$ unit-cells [14].

\section{A. Planar Monopole Antenna over conventional HIS}

In the final structure, a wideband microstrip-fed monopole antenna is integrated with the HIS. After the separate design of both elements, the $3 \times 4$ cell array HIS plane is mounted under the radiating element, as shown in Fig.2 (a). The relative position of the monopole antenna and the HIS plane is illustrated in Fig.2 (b). There are no unit-cells under the feed line of the monopole, because it would deteriorate the feed line transmission characteristics.

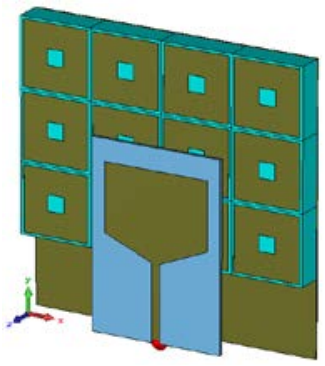

(a)

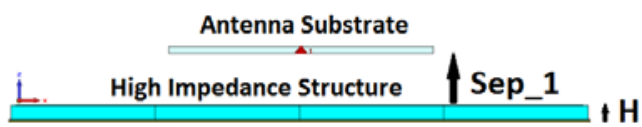

(b)

Fig.2. Monopole antenna over the HIS structure: (a) Front view; (b) Side view with Sep_ $1=0.012 \lambda_{630}, H=0.021 \lambda_{630}$.
After the first simulated results it is observed that the resonant frequency of the resulting design is shifted to higher frequencies. Moreover, the bandwidth of the antenna becomes narrower in comparison to the basic planar monopole antenna. These effects are due to the integration of the HIS plane with the antenna. In order to obtain larger bandwidth, there are two commonly used techniques: First, increasing the substrate relative permittivity, which in this case would lead to a substantial increment in the cost of the HIS structure and thus it is not a viable option for a practical design; second, increasing the thickness of the HIS cell (H parameter in Fig. 2) [11] [15]. Fig. 3 shows the reflection coefficient curves when the thickness $(\mathrm{H})$ of the HIS plane varies from $10 \mathrm{~mm}$ $\left(0.021 \lambda_{630}\right)$ to $40 \mathrm{~mm}\left(0.084 \lambda_{630}\right)$. As shown, the frequency band covered by the optimized HIS plane, with $\mathrm{H}=40 \mathrm{~mm}$ and $\mathrm{L}=115 \mathrm{~mm}$, includes the whole DTV application operating band. However, $\mathrm{H}=40 \mathrm{~mm}$ implies a huge increment in the weight of the HIS substrate, from $1.074 \mathrm{~kg}$ to $4.297 \mathrm{~kg}$, and hence it must be discarded as a viable option for a practical design.

Therefore, in order to keep the same properties of this HIS plane but substantially reducing the weight of the dielectric substrate, the need for a new HIS unit-cell design arises.

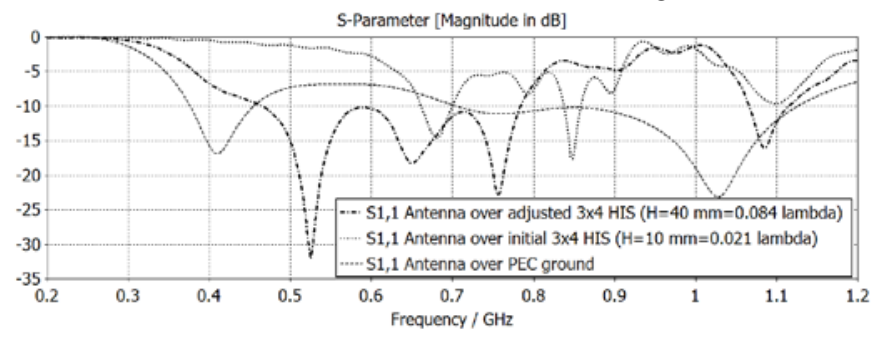

Fig. 3. Simulated reflection coefficient varying the height of the HIS cell $(\mathrm{H}=10 \mathrm{~mm}$ and $\mathrm{H}=40 \mathrm{~mm})$.

\section{LOW-Profile ANTENNA Over A New HIS}

This section proposes a new design for the HIS plane in order to enhance the bandwidth without increasing the substrate thickness, and thus reducing the weight. The new design consists in introducing an air gap between the HIS substrate and its back ground plane as shown in Fig. 4.

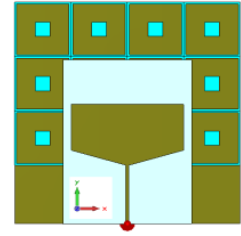

(a)

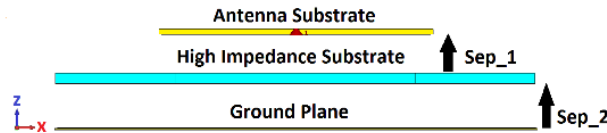

(b)
Fig. 4. Monopole antenna over a new High-Impedance Surface structure: (a) Front view; (b) Side view with Sep_1 $=0.029 \lambda_{630}$, Sep_ $2=0.063 \lambda_{630}$.

\section{A. New HIS Plane Design}

The new HIS plane with air gap (H+Sep_2 thickness) must work in the same AMC in-phase band as the previous unitcell of Sec. II $(\mathrm{H}=40 \mathrm{~mm}$ and $\mathrm{L}=115 \mathrm{~mm})$. After the simulation study, a new cell has been designed with $\mathrm{H}=3 \mathrm{~mm}$ $\left(0.006 \lambda_{630}\right), \mathrm{L}=115 \mathrm{~mm}$, Sep_$\_1=14 \mathrm{~mm}\left(0.029 \lambda_{630}\right)$, Sep_2 $=30$ $\mathrm{mm}\left(0.063 \lambda_{630}\right)$. Total antenna/HIS height (Sep_1+H+Sep_2) is $47 \mathrm{~mm}\left(0.098 \lambda_{630}\right)$, obtaining a low-profile antenna. The 
simulation results of the reflection phase band-gap for this new cell are shown in Fig. 5. As observed, the curve obtained with the new cell $(\mathrm{H}=3 \mathrm{~mm})$ is almost the same as the previous cell case $(\mathrm{H}=40 \mathrm{~mm})$. To analyze the EBG operating frequency band, transmission through the new plane is simulated. A comparative result with the previous plane $(\mathrm{H}=40 \mathrm{~mm})$ is shown in Fig. 6. As observed, the frequency band-gap of the new plane has better behavior than the previous plane, as $S_{12}(\mathrm{~dB})$ presents lower values.

Achieved AMC and EBG properties confirm the excellent results obtained with the use of this new HIS plane. A remarkable reduction in the thickness of the dielectric substrate from $\mathrm{H}=40 \mathrm{~mm}$ to $\mathrm{H}=3 \mathrm{~mm}$ has been achieved, which leads to a substantial weight decrease from $4.297 \mathrm{~kg}$ to $0.352 \mathrm{~kg}$. Total thickness of the HIS structure is $33 \mathrm{~mm}$.

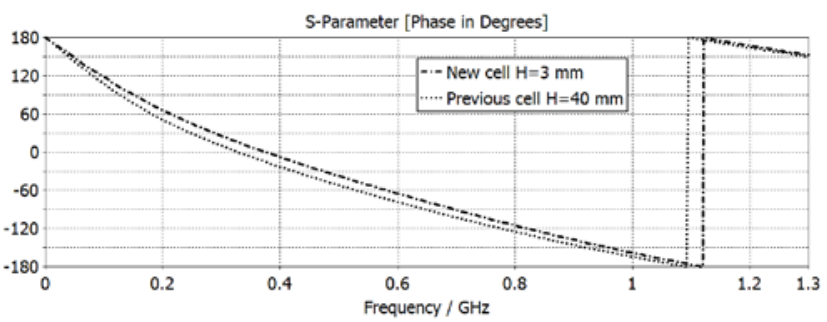

Fig. 5. Comparative of the simulation reflection phase band-gap between the previous cell and the new cell with air gap.

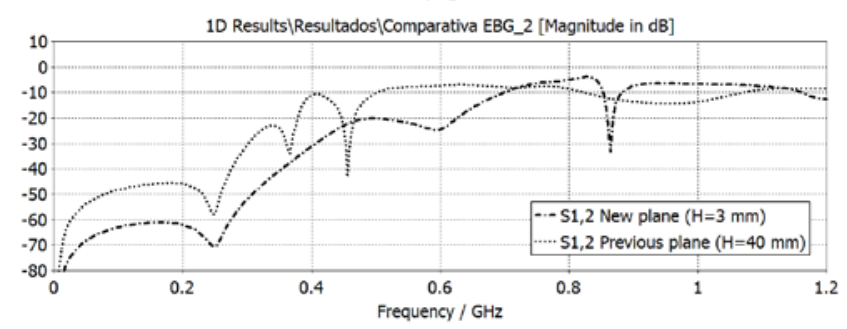

Fig. 6. Simulated band-gap for the new and previous $3 \times 4$ unit-cell.

\section{B. Simulated Results}

The simulated reflection coefficient of the proposed structure with $\mathrm{H}=3 \mathrm{~mm}$ is compared to that obtained using a PEC reflector at the same distance from the antenna, and without using any reflector (simple antenna) in Fig. 7. As it can be observed, the frequency band covered by the monopole antenna over the new HIS plane for $-10 \mathrm{~dB}$ of $\mathrm{S}_{11}$ parameter includes the whole DTV operating band (from $470 \mathrm{MHz}$ to $790 \mathrm{MHz}$ ). This corresponds to a $50.8 \%$ relative impedance bandwidth.

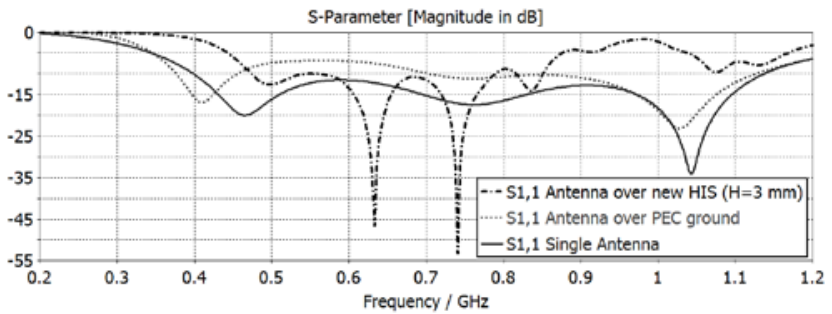

Fig. 7. Simulated reflection coefficient of the antenna over HIS, PEC and without reflector.

\section{Equivalent Circuit Model}

Fig. 8 (a) shows the equivalent circuit model for the final HIS structure. As observed, it can be modeled as a transmission line of length Sep_1+Sep_2 $\left(0.029 \lambda_{630}\right.$ $+0.063 \lambda_{630}$ ) loaded with a parallel reactance (in this case, a capacitance of $\mathrm{C}=2.58 \mathrm{pF}$, obtained with AWR software tuning tool). Fig. 8 (b) shows a comparison of the phase of the reflection coefficient for the proposed HIS structure and for the presented circuit model. A complete coincidence of both curves can be noticed.

The equivalent circuit model shows that the parallel reactance loading allows to decrease the distance to the reflector from $\lambda_{630} / 4$ to $0.092 \lambda_{630}$.

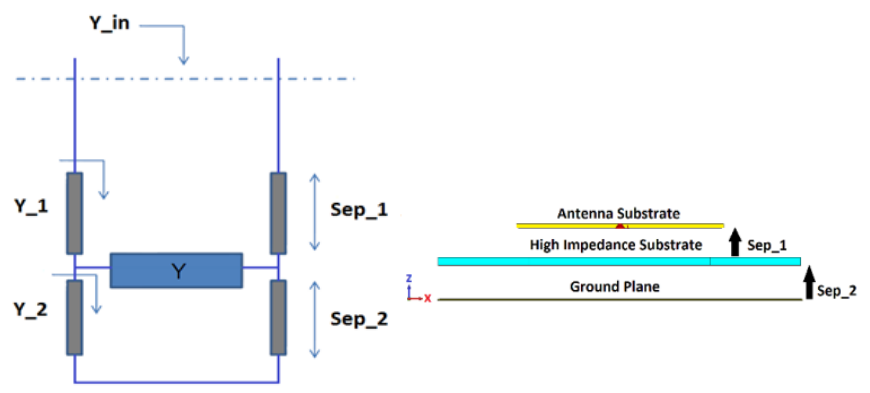

(a)

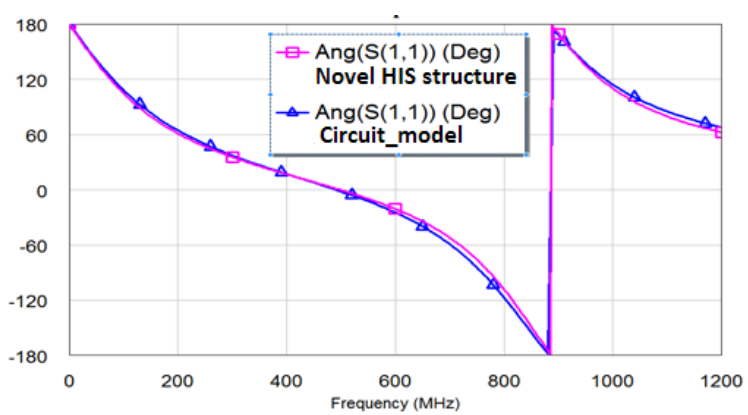

(b)

Fig. 8. Characterization of the new HIS structure. (a) Circuit model, (b) Comparison of the reflection coefficient phase.

\section{Measurement Results of the Antenna Prototype}

In this section, the fabricated prototype and the measurement results are presented. Fig. 9 shows the manufactured prototype, in which nylon separator elements are employed to set the relative distances Sep_1 and Sep_2, shown previously in Fig. 4.

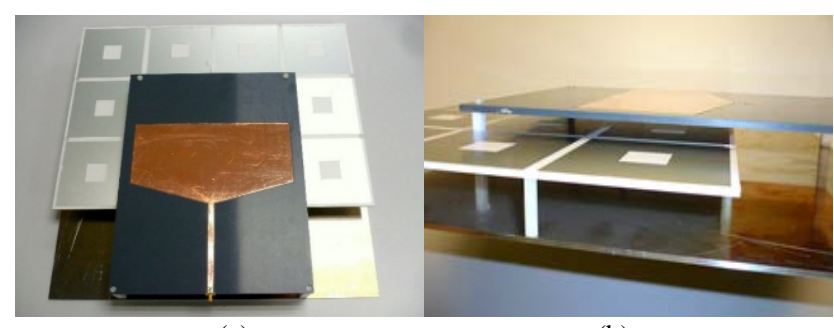

(a)

(b)

Fig. 9. Manufactured prototype: (a) Top view; (b) Lateral view.

\section{A. S-Parameters and Radiation Patterns}

The simulated and measured $S$-parameters of the prototype antenna are shown in Fig. 10. It can be observed that the measured -10 dB impedance bandwidth ranges from $480 \mathrm{MHz}$ to $850 \mathrm{MHz}$, corresponding to a $55.6 \%$ relative bandwidth. That supposes an increase of $4.8 \%$ compared to simulation. 
Measured 2D radiation patterns at $\mathrm{XZ}$ and $\mathrm{YZ}$ planes for the proposed antenna at three different frequencies $(470 \mathrm{MHz}, 630$ $\mathrm{MHz}$ and $790 \mathrm{MHz}$ ) within the operating band are presented in Fig. 11. It can be observed that, as desired, directional radiation behavior is obtained for all frequencies, due to the use of the HIS reflector. Some degradation of the radiation pattern is observed in the YZ-plane at $790 \mathrm{MHz}$, since this is near the upper frequency limit of EBG behavior of the HIS structure. The measured gain (reaching up to $10.25 \mathrm{dBi}$ ) and the radiation efficiency (reaching 97\%) are presented together with the directivity in Fig. 12.

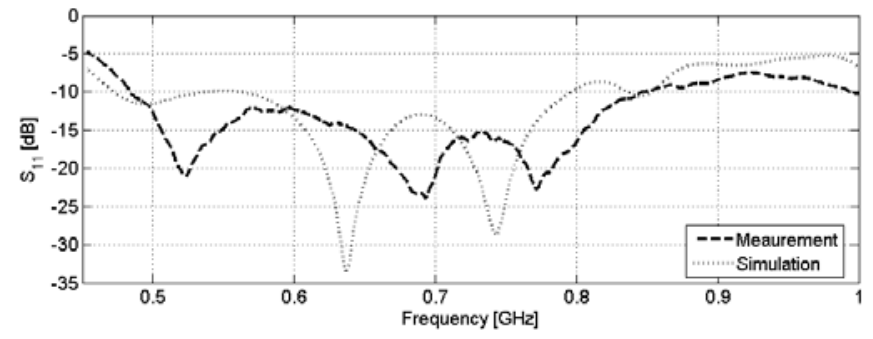

Fig. 10. Simulated and measured reflection coefficient (dB).

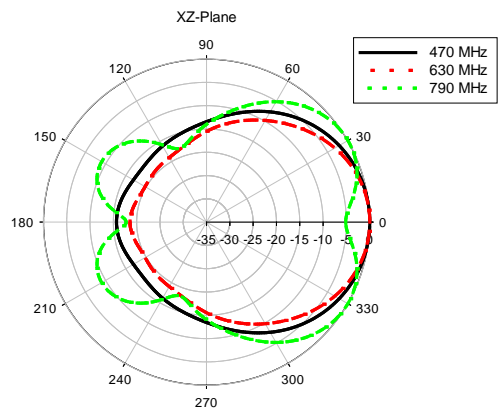

(a)

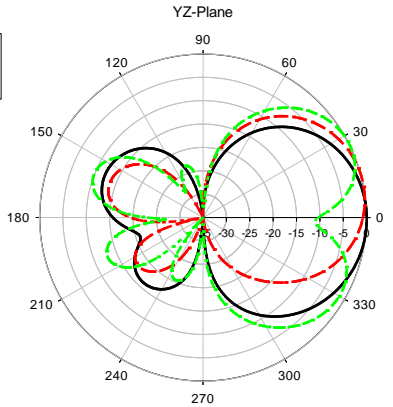

(b)
Fig. 11.Measured radiation patterns at different frequencies (a) in the XZplane; (b) in the YZ-plane.

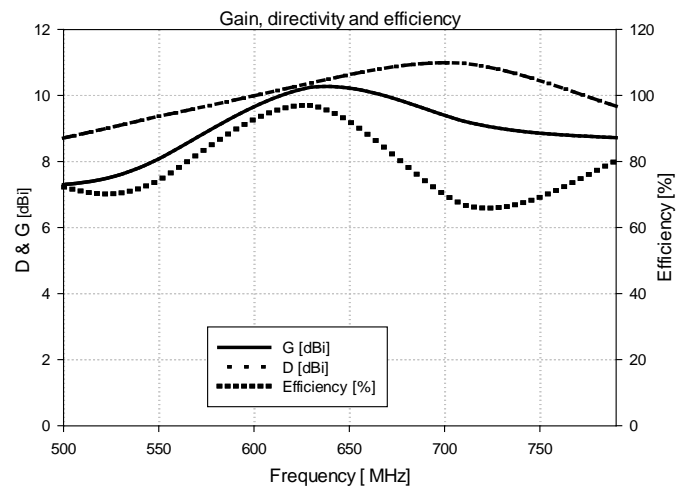

Fig.12. Measured gain, directivity and efficiency.

\section{V.CONCLUSION}

A novel wideband, light weight and very low profile antenna (with a height lower than $0.1 \lambda_{630 \mathrm{MHz}}$ ) has been proposed for DTV applications in the UHF band. A HIS plane consisting of a FSS, an air gap and a ground plane is used as a reflector for the antenna in order to obtain a lightweight structure. The antenna prototype (with a single radiating element) achieves $55.6 \%$ relative bandwidth, high gain of
$10.25 \mathrm{dBi}$ and a radiation efficiency of $97 \%$. With the use of the proposed reflecting structure a peak gain improvement of 7 $\mathrm{dBi}$ with respect to the simple monopole and $2 \mathrm{dBi}$ above the traditional HIS structure (without air gap) is obtained. Moreover, a significant weight reduction has been achieved compared to traditional solutions using HIS, which is essential for practical applications. The proposed antenna happens to provide a planar and more compact solution than traditional Yagi-Uda antennas for digital television reception. Future work is focusing on analyzing the use of a multi-layer FSS [13] as a reflector.

\section{REFERENCES}

[1] D. Sievenpiper, L. Zhang, R. F. Jimene Broas, N. G. Alexopolous, and E. Yablonovitch, "High-impedance electromagnetic surfaces with a forbidden frequency band,” IEEE Trans. Microw. Theory Tech., vol. 47, no. 11, pp. 2059-2047, Nov. 1999.

[2] Y. Rahmat-Samii, H. Masallaei, "Electromagnetic bandgap structures: classification, characterization and applications," $11^{\text {th }}$ Int. Conf. on Antennas and Propag., no. 480, pp. 560-564, April 2001.

[3] G. Goussetis, A.P. Feresidis, and J.C. Vardaxoglou, "Tailoring the AMC and EBG characteristics of periodic metallic arrays printed on grounded dielectric substrate”, IEEE Trans. Antennas Propag., vol. 54, no. 1, pp. 82-89, Jan. 2006

[4] A. P. Feredisi, G. Goussetis, S. Wang, and J. C. Vandaxoglou, "Artificial magnetic conductor surfaces and their application to lowprofile high-gain planar antenas”, IEEE Trans. Antennas Propag., vol. 53, no. 1, pp. 209-215, Jan. 2005.

[5] F. Yang and Y. Rahmat-Samii, "Reflection phase characteristics of the EBG ground plane for low profile wire antennas," IEEE Trans. Antennas Propag., vol. 51, no. 10, pp. 2691-2703, Oct. 2003.

[6] K. Agarwal, Nasimuddin, and A. Alphones, "Wideband circularly polarized AMC reflector backed aperture antenna", IEEE Trans. Antennas Propag., vol. 61, no. 3, pp. 1456-1461, March 2013.

[7] W. Yang, H. Wang, W. Che, and J. Wang, "A wideband and high-gain edge-fed patch antenna and array using artificial magnetic conductor structures”, IEEE Antennas Wireless Propag. Lett., vol. 12, pp. 769-772, 2013.

[8] B. S. Cook, and A. Shamim, "Utilizing wideband amc structures for high-gain inkjet-printed antennas on lossy paper substrate," IEEE Antennas Wireless Propag. Lett., vol. 12, pp. 76-79, Jan. 2013.

[9] P. Prakash, M.P. Abegaonkar, A. Basu, and S.K. Kou, "Gain enhancement of a CPW-fed monopole antenna using polarizationinsensitive AMC structure”, IEEE Antennas Wireless Propag. Lett., vol. 12, pp. 1315-1318, 2013.

[10] R. Cosmina Hadarig, M.E. de Cos, and F. Las-Heras, "UHF dipoleAMC combination for RFID applications", IEEE Antennas Wireless Propag. Lett., vol. 12, pp. 1041-1044, 2013.

[11] S.R. Best and D.L Hanna, "Design of a broadband dipole in close proximity to an EBG ground plane", IEEE Antennas Propag. Mag., vol. 50, no. 6, pp. 52-64, Dec. 2008.

[12] M. Pasian, S. Monni, A. Neto, M. Ettorre and G. Gerini, "Frequency Selective Surfaces for Extended Bandwidth Backing Reflector Functions”, IEEE Trans. Antennas Propag., vol. 58, no.1, pp. 43-50, January 2010.

[13] Y. Ranga, L. Matekovits, K.P. Esselle, and A.R. Weily, "Multioctave frequency selective surface reflector for ultra-wideband antennas," IEEE Antennas Wireless Propag. Lett,.vol. 10, pp. 219-222, 2011.

[14] N. Mohamed Mohamed-Hicho, E. Antonino-Daviu, M. Cabedo-Fabrés, and M. Ferrando-Bataller, "A Novel Low Profile High-Gain UHF Antenna Using High-Impedance Surfaces”, $8^{\text {th }}$ European Conference on Antennas and Propagation (EuCAP2014), April 2014.

[15] M. Mantash, A. C. Tarot, S. Collardey, and K. Mahdjoubi, "Dual-band CPW-fed g-antenna an EBG structure", 2010 Loughborough Antennas and Propag. Conference (LAPC), pp. 453-456, November. 2010. 